\title{
GUIDING THE GUIDERS: FOUNDATIONS OF A MARKET-DRIVEN THEORY OF DISCLOSURE
}

\author{
M. GIETZMANN \\ Department of Accounting, Bocconi University \\ Via Roberto Sarfatti 25, 20100 Milano, Italy \\ E-mail: miles.gietzmann@unibocconi.it \\ A. J. OSTASZEWSKI \\ Mathematics Department, London School of Economics \\ Houghton Street, London WC2A 2AE, UK \\ E-mail: a.j.ostaszewski@lse.ac.uk \\ M. H. G. SCHRÖDER \\ Keplerstrasse 30, D-69469 Weinheim, Germany \\ E-mail: maths.mhgsch@gmail.com
}

\begin{abstract}
A foundational approach is developed for a mathematical theory of managerial disclosure in relation to asset pricing; this involves both the earnings guidance disclosed by firm management and market 'trackers' pricing the firm's exposure to quotable risks.
\end{abstract}

1. Introduction. Asset-pricing models make assumptions about how information arrives and is disclosed to its investors (henceforth the market). For assets arising out of a productive activity by a firm, management reports based on internal audits of the various accounting numbers (accounting variables), prepared in time for the scheduled (publicly pre-specified) dates, are one such source. If, by the nature of their activity, management

2010 Mathematics Subject Classification: Primary 91G50; Secondary 91G80, 93E11, 93E35, 60G35, 60G25.

Key words and phrases: risk-neutral valuation, asset-price dynamics, earnings guidance, optimal censoring, materiality, state observer system.

The paper is in final form and no version of it will be published elsewhere. 
make more frequent audits (for instance in directing replenishment to a specific level, which enforces frequent stock-taking, as in the retail business), then opportunities arise for 'early' (unscheduled) disclosure. How should this additional information be used to signal the firm's superior value, i.e. to upgrade its share-price? When (or how) should the market 'price in' the absence of early disclosures by a firm to include the possibility that no news is bad news. The answer must rely on an equilibrium between the market's ability to down-grade the share-price and the firm's ability to take advantage of ignorance: hiding some bad news within the uncertain cause of absent news, i.e. censoring the information.

The accounting literature has usually approached this question by including a specified (i.e. known in advance), single, 'additional' interim reporting date, ahead of the next scheduled disclosure, and allowing for absence of an early disclosure by randomizing the possibility that management has held an additional audit — see Dye, JunK. However, with the early date a datum, this approach places limits on comparative analysis; for an equally spaced multi-period model see [EinZ].

1.1. Earnings guidance: strategic considerations. In this the companion paper to GieO we propose an alternative general approach, by instilling more realism into the stylized Black-Scholes model of [GieO]. There the market determination of the (share-)price of the firms in a sector reflects only the discretional information strategically released by a firm (i.e. with anticipation of its price effect), usually in the form of earnings guidance, as below, at a stochastic time-point (i.e. at unknown dates in advance of a subsequent mandatory date of information disclosure). Despite this highly specific origin for the arrival of information in the market, that model holds considerable advantages, thanks to its continuous-time approach which overcomes the limitations of the traditional 'two-period analysis' just mentioned. There unspecified moments in time offer an early disclosure. By way of an example from [GieO], which goes beyond the scope intended here, there is a derivable 'band-wagon' effect whereby the introduction of multiple sources of information reduces an individual firm's optimal frequency of disclosures by reference to time left to the next mandatory disclosure date.

Typically, however, the market responds also to other public sources of information, such as trading in the shares of the firm, and by assessing the exposure of the firm to such economic risks as may be priced by market-quoted options.

Here we create a more general framework to include such other, already existing, market-based information enabling the market to make proper use of this additional information about the firm. This prompts a deeper analysis, equivalently a formalization at a foundational level, of the various mechanisms at work, offered in the Complements Section. For simplicity, we consider here only the situation where the market's concern is for a single firm, rather than a whole sector. In this we are guided by the clarifying simplifications that occur in the case of an isolated ('single') firm in the stylized model GieO].

There the firm itself comes to know ('observes') its own state $V_{t}$ only at discrete, stochastically generated times $t$, not known to the market; the manager, occupied by a variety of tasks, cannot receive observations except when these breach agreed thresholds, as reported by personnel delegated to collect this information, perhaps continuously. This 
feature of a hidden observation scheme enables the firm to bury (hide) 'bad' news and only report 'sufficiently good' news, principally because the manager cannot at any time credibly claim as absent an observation at that time. That model prices the firm in periods of silence, i.e. when the firm fails to supply an early report of information. Key to this is identifying at each time $t$ a value $L_{t}$ such that if $L_{t}$ happened to coincide with the true and currently observed state $V_{t}$, the firm would be indifferent, as regards its market valuation (i.e. given the market's information ex ante), between choosing to disclose or to withhold the current observed state $V_{t}$. Such an indifference level $L_{t}$, determined by the information available from before time $t$, is typically unique. Censoring, i.e. suppression of an observation below this unique $L_{t}$, draws from the market a valuation of the firm at $L_{t}$. In fact, $L_{t}$ is the largest possible valuation of the firm, consistent with the information available from before time $t$. As such it is termed the optimal censor of time $t$. Note that observations above $L_{t}$ that are disclosed cause an upward jump in the firm's valuation. We should emphasize that only truthful disclosures are allowed in the model.

The mathematical argument is based on risk-neutral valuation, which must incorporate the potential future re-evaluations of firm-value consequent upon future early disclosures.

The existence of an indifference pricing process is directly attributable to the firm knowing the market's filtration $\boldsymbol{F}^{*}=\left\{\mathcal{F}_{t}^{*}\right\}_{t}$ and the mechanics of how the market performs computations based on past disclosures (in particular, the probabilities at each instant which the market attaches to the firm suppressing an observation of its state). Since the firm's filtration $\boldsymbol{F}=\left\{\mathcal{F}_{t}\right\}_{t}$ is an enlargement of $\boldsymbol{F}^{*}$ [Jeu], in that the firm feeds the market with information by choosing when to supply its private observations, one may say that the firm emulates (can simulate) the market. In turn the market's calculations are based on the firm's algorithm, though not on the firm's up-to-date observations. The indifference price arises from characterizing a notional parametric equilibrium between the two agents: the firm and the market (we do not differentiate between informed and noisy traders), selecting parameters in the computation they use to second-guess each other.

The paper identifies the mechanisms underlying some fairly general valuation procedures, allowing the market to form its beliefs from additional information and the firm to exploit the market beliefs by disclosing value superior to that belief, but nevertheless to give a fair view of future disclosures. As these mechanisms are inspired by the principal argument and findings of [GieO], we close here with a summary of that argument (in the simplified notation used below). Suppose the next mandatory disclosure is at the terminal time 1 and that, under the market's risk-neutral measure $Q$, at time $t<1$ the probability of a disclosure to the market occurring at time $T \in(t, 1]$ is $\tau_{T}$. (In [GieO] opportunities to observe the state of the firm are generated by a Poisson clock.) Based on its information at time $T$, the earnings guidance announced at that time by the firm gives its target terminal value as $V T=E^{Q}\left[V_{1} \mid \mathcal{F}_{T}\right]$; the target and the two filtrations above are related to the indifference level $L_{T}$ of time $T$ by the two equations

$$
\begin{gathered}
V T=\tau_{T} E^{Q}\left[V_{1} \mid V_{T} \geq L_{T}, \mathcal{F}_{T}\right]+\left(1-\tau_{T}\right) L_{T}, \\
L_{T}=E^{Q}\left[V_{1} \mid N D_{T}\left(L_{T}\right), \mathcal{F}_{T}^{*}\right] .
\end{gathered}
$$

Here $N D_{T}(L)$ is the event that no disclosure occurs at time $T$, which means that either 
there has been no opportunity to observe $V_{T}$ or else the manager has observed $V_{T}$ but $V_{T} \leq L$. From here, in the context of [GieO], given how $\mathcal{F}_{T}^{*}$ is generated from $\mathcal{F}_{T}$ via $\mathcal{F}_{T}^{*}$-measurable decisions, one deduces in the limit as $T \rightarrow t$ from (1.1.1a) that $t \mapsto L_{t}$ satisfies a simple ordinary differential equation (involving the instantaneous variance of $Q$ and the Poisson clock's intensity). Assuming multiplicative scalability, that $V_{T+u}=V_{T} \tilde{V}_{u}$ with independence of $V_{T}$ and $\tilde{V}_{u}$, equations (1.1.1) can be further simplified.

In summary, apart from simplifications, this paper's contributions include: announcements of both sufficiently good and additionally of sufficiently bad news (dual, 'materiality' aspects in the release of private information), incorporation of current public information in modelling market sentiment, and comparative statics of early disclosures.

The paper is organized as follows. Section 2 contains a preliminary discussion of our modelling aims. Section 3 models the market's beliefs as to firm value, based on expected performance indices and is supported by a geometric Brownian (GMB) implementation. This is followed in Section 4 by a model of the firm setting its target values; using a benchmark scheme to be followed by the firm in observing its own state, this is shown at its simplest to be similar to determining option exercise values, and is supported by an indicative GMB implementation. This enables us to perform comparative statics in Section 5, which we conclude meets a primary objective: to show how parameter values determine early or delayed voluntary release of information in equilibrium. We comment briefly on the implications of our approach in Section 6 , thus rounding off the paper's contribution, and close in Section 7 with Complements indicating a framework for generalizations and potential variations. An Appendix collects and reviews GMB formulas pertinent for the paper.

2. Model preliminaries. In this section we introduce a model of how a firm $F$ decides to disclose information intermittently at times $t$ to the market $M$ about its state $V_{t}$, voluntarily between legally mandated (mandatory) disclosure dates. This involves modelling how the market forms beliefs in periods of silence about the true current value $V_{t}$; this aspect will be captured by $V_{t}^{*}$, the market's proxy for $V_{t}$. (We regard the market as dual to the firm, hence the 'star' notation here and below.)

Our first two tasks are: to model the beliefs of $M$ (in Section 3) and then to model $F$ 's choice of 'equilibrium' indifference level (in Section 4), below which an observation of $V_{t}$, if any, is not disclosed (as in the Introduction). We will rely on tractable BlackScholes frameworks, and in the second task we will be guided by the findings of [GieO]. In Section 5 we prove the existence of these indifference levels in a benchmark context. We may then pass to calculations which will yield conclusions, in particular, about the likelihood of early disclosure. This enables us in Section 6 to address comparative statics of voluntary (i.e. early) disclosures, matters beyond the reach of [GieO].

Thereafter, we discuss generalizations identifying potential for more sophisticated models (e.g. inclusion of analyst forecasts).

The firm has 'private' access, according to some observation scheme - possibly also intermittent - to its 'state' $V_{t}$ (thought of as the income stream). This is modelled as a random time $\tau$ not known to $M$. The firm applies fixed decision rules by which it determines at time $t$ whether to withhold any observation it may have, or to disclose its 
information voluntarily (and truthfully) to the market via two items of information: (i) the current value $V_{t}$, and (ii) the expected state at the terminal date, i.e. the next mandatory disclosure date. We term the former the declared current value $V C$ and the latter its declared target value $V T$ (current as at the date of its disclosure). The firm's intention is to achieve the highest possible market valuation at each point in time; here this is implemented by use of a fixed decision rule, based both on its own private information about its state and on the market's public belief about the state of the firm, which in turn depends on the market's information base. We term this, publicly held belief about the value, the market sentiment. $F$ forms its expectations by reference to a measure $Q_{V T}$ (labelled by the last declared target) under which its (discounted) observation process is a martingale.

To model market sentiment, we borrow and amend a concept from control theory, that of a state observer (a.k.a. 'state estimator') system; for a discussion see Section 7.1(iv).

In the GieO model the observation scheme was a Poisson clock with known jump intensity; the market sentiment was an equilibrium valuation obtained from the latest disclosed state-value, prudently discounted downwards (i.e. by incorporating the possible undisclosed poor performance); discounting is by a rate determined by the (known) Poisson jump intensity.

Below, $V_{t}^{*}$, the market's proxy for $V_{t}$, gives expression to this market sentiment. This proxy will typically be in terms of the latest disclosed information and the current value of some fixed portfolio of traded assets with value $S_{t}^{*}$ (e.g. current value, average value, record value to date). The portfolio, termed the tracker, is viewed by the market as capturing the firm's exposure to quotable (market-priced) risks. The key property of $S_{t}^{*}$ is that it is priced by a risk-neutral (i.e. market) measure. That is, $M$ forms its expectations by reference to a measure $Q^{*}$ under which the (discounted) tracker process is a martingale. This measure is connected with that of $F$; at each time $t$, it is the case that $F$ has access to $M$ 's information $\mathcal{F}_{t}^{*}$; hence, its own filtration $\left\{\mathcal{F}_{t}\right\}_{t}$ is an enlargement of $\left\{\mathcal{F}_{t}^{*}\right\}_{t}$, and this implies

$$
Q^{*}\left|\mathcal{F}_{t}^{*}=Q_{V T}\right| \mathcal{F}_{t}^{*} .
$$

As in [GieO], so too here, the link between market sentiment as expressed by $V^{*}$ and asset $S^{*}$ needs to be determined by equilibrium considerations: if at time $t$ the firm applies a decision rule $h$ to the observations of $S^{*}$ and $V$, it will determine an indifference level $L$ (as in the Introduction) which, if $F$ observed that $V_{t}=L$, would make $F$ indifferent between disclosure or otherwise of $V_{t}$. While the firm determines its disclosure using a rule $h$ (below) that exploits any superiority of the observed value over market sentiment, rather than its expected terminal value, it complements such a disclosure by supplying the market with information about the expected terminal value.

We take the decision rules for $F$ and consequently also for $M$ (with starred notation for the latter's rules) in the (time-independent) form

$$
h_{\varepsilon, a}(t, x, y)=(x-(1+a) y) \varepsilon, \quad(x, y \in \mathbb{R}, t>0)
$$

where $a>-1$ is termed a mark-up, and $\varepsilon= \pm 1$ its signature, positive when deciding a good-news disclosure event at time $u$, say, when

$$
V_{u} \geq(1+a) V_{u}^{*},
$$


and respectively negative for a disclosure of bad news. (A dynamic variant is considered briefly in Section 7.1.) These may be viewed as backed by a theoretical justification for such a 'principal-agent' delegation (here a shareholder-mandated policy): see the classic paper [BaiD, Proposition 1.4] for a rigorous derivation of control limits, using an 'evaluation and control' method. The argument there refers to the costs versus the benefits of extracting information and the authors claim support of (perhaps, anecdotal) hard evidence that such rules are observed in practice.

We close by stressing that the various asset-price dynamics here are modelled only between consecutive disclosures — in 'periods of silence'.

3. Market sentiment: shadowing the firm. For the first of the two tasks we identified in Section 2, the modeling of market sentiment via $V^{*}$, we focus on the period of time between two consecutive dates for mandatory disclosure, which is formalized as the (open) time interval $\left(T_{0}, T_{1}\right)$, such that the (firm) $F$ has disclosed at $T_{0}$ two items of data: current state $V C$ and declared target $V T$. At each of point $T$ during this period, $F$ has to decide on voluntary disclosure. We make this decision take into account also the time- $T$ value of some observation variable that gives expression to publicly available information; we call this value $X_{T}$. Here, to fix ideas, we consider the simple but typical case where $X$ coincides with the market's proxy for $V$, choosing $X=V^{*}$.

Disclosure will occur at time $T$ if we have

$$
h\left(T, V_{T}, V_{T}^{*}\right) \geq 0 ;
$$

here $F$ applies its decision rule $h$, taken in general to depend on the time- $T$ values of $V$ and $V^{*}$, and possibly on $T$ itself (a possibility ruled out below to simplify calculations, hence the rules in Section 2 above).

To explain what happens when there is a (first) time $T$ where disclosure occurs, write $T_{+}$for this time. Then, firstly, $F$ will declare $V_{T_{+}}$as its (new) current state $V C$ and set a (new) target value $V T$. Secondly, the above monitoring for voluntary disclosure will re-start now on the period of time between $T_{0}=T_{+}$and $T_{1}$, the next date of mandatory disclosure after $T_{+}$.

It remains to devise justifiable models for, firstly, the market's proxy process $V^{*}$, which we will view below as 'shadowing the firm', and, secondly, for the targets $V T$, whose construction in Section 4 applies actuarial principles.

3.1. Two modelling assumptions. We begin by identifying how to model $V_{t}^{*}$. This will be determined by two components. Although the entire process is driven by a specified market portfolio $S^{*}$, the tracker, nevertheless, at each non-disclosure time-point $t$ in $\left(T_{0}, T_{1}\right)$ a correction term needs to be included to price in the effects of any possible future disclosure event, say of time $\tau$. We thus aim for a two-component model

$$
V_{t}^{*}=S_{t}^{*}+\Delta V_{t}^{*}
$$

which requires that we model $\Delta V_{t}^{*}$. The latter must refer to contingent claims in regard to whether or not $F$ has attained its target to date; with $h^{*}$ a market decision rule, 
this requires pricing contingent claims on $[t, \tau]$ characterized by the instantaneous time- $\tau$ pay-off

$$
S_{\tau}^{*} \mathbf{1}_{\left\{h^{*}\left(\tau, S_{\tau}^{*}, V T\right) \geq 0\right\}}, \quad \text { for } \quad \tau=\tau_{V T} .
$$

So this is a 'securitization' similar to standard derivative instruments. It depends on a 'random time' $\tau=\tau_{V T}$ (with values in $\left(t, T_{1}\right]$ ) unknown to the agent $M$. Unfortunately, pricing these depends on individual investor attitudes, so on micro level information, typically unobservable. The pragmatic approach is to replace the pricing of these claims with approximations. Assuming a constant risk-free rate $r$ in force, we now make explicit a first modelling assumption, that with $\tau=\tau_{V T}$

$$
\Delta V_{t}^{*}:=\exp \left(-r\left(T_{1}-t\right)\right) E^{Q^{*}}\left[S_{\tau}^{*} \mathbf{1}_{\left\{h^{*}\left(\tau, S_{\tau}^{*}, V T\right) \geq 0\right\}} \mid \mathcal{F}_{t}^{*}\right]-S_{t}^{*}
$$

here $Q^{*}$ is an assumed risk-neutral measure conditional on market information - conditional at time $t$ on market information $\mathcal{F}_{t}^{*}$ (with the expectation on the right assumed meaningful). The formula identifies $\Delta V_{t}^{*}$ as the excess over $S_{t}^{*}$ of the fair value of the effect of a disclosure occurring at time $\tau$. The final step at time $t$ is to pass to an approximation, which make explicit a second modelling assumption, that

$$
S_{\tau}^{*} \mathbf{1}_{\left\{h^{*}\left(\tau, S_{\tau}^{*}, V T\right) \geq 0\right\}} \approx E_{t}^{*} \mathbf{1}_{\left\{h^{*}\left(t, \Sigma_{t, T_{1}}^{*}, V T\right) \geq 0\right\}} .
$$

Here, on the one hand, $\Sigma_{t, T_{1}}^{*}$ is some chosen tracker-performance index over the entire remaining time interval, for instance

$$
\left.\begin{array}{l}
\Sigma_{t, T_{1}}^{*}=\max \left\{S_{u}^{*} \mid u \in\left[t, T_{1}\right]\right\}, \quad \text { or } \\
\Sigma_{t, T_{1}}^{*}=1 /\left(T_{1}-t\right) \int_{\left[t, T_{1}\right]} S_{u}^{*} \mathrm{~d} u, \quad \text { or } \\
\Sigma_{t, T_{1}}^{*}=\min \left\{S_{u}^{*} \mid u \in\left[t, T_{1}\right\} ;\right.
\end{array}\right\}
$$

and, on the other hand, a typical choice for $E_{t}^{*}$ is obtained by solving $h^{*}\left(t, E_{t}^{*}, V T\right)=0$. This last has a unique solution, the result of the simple form of decision rule in Section 2. Thus in good-news situations $E_{t}^{*}$ identifies a value at-least-as-good-as the true value $S_{\tau}^{*}$ at disclosure; in bad-news situations such an $E_{t}^{*}$ will be at-most-as-bad-as that. We summarize these modelling considerations in:

Proposition 3.1. The two modelling assumptions (3.1.3), (3.1.4) imply that

$$
V_{t}^{*}=\left(S_{t}^{*}+\Delta E_{t}^{*}\right) Q^{*}\left(h^{*}\left(t, \Sigma_{t, T_{1}}^{*}, V T\right) \geq 0 \mid \mathcal{F}_{t}^{*}\right) \exp \left(-\left(T_{1}-t\right) r\right),
$$

where:

(i) $S^{*}$ is assumed to start with the value $V C$ at time $T_{0}$,

(ii) $\Delta E_{t}^{*}=E_{t}^{*}-S_{t}^{*}$, and

(iii) $\Sigma_{t, T_{1}}^{*}$ is chosen as in example (3.1.5) above.

3.2. A geometric Brownian implementation. On the firm side we take the firm's observation process $V$ to be modelled by a geometric Brownian motion $X$ :

$$
X_{s+t}=X_{t} \exp \left(\left(\mu_{V T}-\frac{1}{2} \sigma_{V T}^{2}\right) s+\sigma_{V T} W_{V T, s}\right), \quad s \in[0, T-t]
$$

here $W_{V T}$ is a Brownian motion independent of time- $t$ information $\mathcal{F}_{t}$, with $\sigma_{V T}>0$ and $\mu_{V T} \in \mathbb{R}$ fixed. 
Likewise, on the market side we adopt a Black-Scholes model with one risky security $S^{*}$, so modelled again by a geometric Brownian motion; this specification satisfies the requirements of Proposition 3.1 and carries several advantages beside: firstly, modelcompleteness (see for example [MusR, Proposition 8.2.1, p. 302] for the completeness of the multi-dimensional model) and, secondly, the ability of being re-started with value $V C$ at time $T_{0}$. We take the dynamics in the form

$$
S_{t+u}^{*}=S_{t}^{*} \exp \left(\mu^{*} u+\sigma^{*} W_{u}^{*}\right), \quad u \in[0, \infty),
$$

with Brownian motion $W^{*}$ independent of time- $t$ information $\mathcal{F}_{t}^{*}$, and two parameters: volatility $\sigma^{*}>0$ and drift $\mu^{*}=r-\delta-(1 / 2) \sigma^{* 2}$, for some $\delta \in \mathbb{R}$.

This modelling choice ensures that the probabilities in the formula of Proposition 3.1 are well-defined (see below), emerging as tail probabilities for good-news decisions, since

$$
h^{*}\left(t, \Sigma^{*}, V T\right) \geq 0 \quad \text { iff } \quad h_{+1, a^{*}}\left(\Sigma^{*}, V T\right) \geq 0 \quad \text { iff } \quad \Sigma^{*} \geq\left(1+a^{*}\right) V T,
$$

for $\Sigma^{*}$ any random variable. We will provide explicit formulae for these in the next subsections. We focus here on the choices of $(3.1 .5 \mathrm{a}, \mathrm{c})$ of max- and min-tracker-performance indices $\Sigma_{t, T_{1}}^{*}\left(S^{*}\right)$, leaving aside the average value index.

3.2.1. $V^{*}$ from the running-max approximation. We first deal with the running-max

$$
\Sigma^{*}:=\max \left\{S_{u}^{*} \mid u \in\left[t, T_{1}\right]\right\} .
$$

We note a reversion from bad-news to good-news decisions via:

$$
Q^{*}\left(h_{+1, a^{*}}\left(\Sigma^{*}, V T\right) \geq 0\right)+Q^{*}\left(h_{-1, a^{*}}\left(\Sigma^{*}, V T\right)>0\right)=1 .
$$

The actual influence of the second term on the first is determined by the relative size of $\left(1+a^{*}\right) V T$ and $S_{t}^{*}$, so according to the sign of

$$
A^{*}=\log \left(\left(1+a^{*}\right) V T / S_{t}^{*}\right) .
$$

Below Erfc is the complementary error function, for which see Section A.2 of the Appendix. Equations (A.7a) and (A.7b) in the Appendix yield the following results.

Proposition 3.2. If $A^{*} \leq 0$, equivalently, $S_{t}^{*} \geq\left(1+a^{*}\right) V T$, then

$$
Q^{*}\left(h_{-1, a^{*}}\left(\Sigma^{*}, V T\right)>0\right)=0,
$$

and there is no 'bad-news' influence; thus

$$
Q^{*}\left(h_{+1, a^{*}}\left(\Sigma^{*}, V T\right) \geq 0\right)=1, \quad \text { if } S_{t}^{*} \geq\left(1+a^{*}\right) V T .
$$

If $A^{*}>0$, equivalently $S_{t}^{*} \leq\left(1+a^{*}\right) V T$, then 'bad-news' carries influence measured by

$$
Q^{*}\left(h_{+1, a^{*}}\left(\Sigma^{*}, V T\right) \geq 0\right)=1-Q^{*}\left(h_{-1, a^{*}}\left(\Sigma^{*}, V T\right)>0\right),
$$

and we have

$$
\begin{aligned}
& Q^{*}\left(h_{+1, a^{*}}\left(\Sigma^{*}, V T\right) \geq 0\right) \\
& \quad=\frac{1}{2} \operatorname{Erfc}\left(\frac{A_{t}^{*}-(T-t) \mu^{*}}{\sigma^{*} \sqrt{2(T-t)}}\right)+\frac{1}{2} \exp \left(\frac{2 \mu^{*} A_{t}^{*}}{\left(\sigma^{*}\right)^{2}}\right) \operatorname{Erfc}\left(\frac{A_{t}^{*}+(T-t) \mu^{*}}{\sigma^{*} \sqrt{2(T-t)}}\right) .
\end{aligned}
$$


To complete the picture, note that the bad news scenario when $\Sigma^{*}$ is the runningmaximum can be read back from:

$$
Q^{*}\left(h_{-1, a^{*}}\left(\Sigma^{*}, V T\right) \geq 0\right)=0, \quad \text { if } S_{t}^{*}>\left(1+a^{*}\right) V T,
$$

and that for $A^{*}>0$ this probability is given by equation $(3.2 .4 \mathrm{c})$ above.

3.2.2. $V^{*}$ from the running-min approximation. We now deal with running-min

$$
\Sigma^{*}:=\min \left\{S_{u}^{*} \mid u \in\left[t, T_{1}\right]\right\} .
$$

The good-news and bad-news formulas hold good and, viewed technically, may be derived by replacing $\mu^{*}$ by $-\mu^{*}$ and $A^{*}$ by $-A^{*}=\log \left(S_{t}^{*} /\left(\left(1+a^{*}\right) V T\right)\right)$; and switching to probabilities complementary to those in (3.2.1): see the discussion for equation (A.8) in Section A.2 of the Appendix. From there, we have explicitly:

Proposition 3.3. If $S_{t}^{*} \leq\left(1+a^{*}\right) V T$, then

$$
\begin{aligned}
& Q^{*}\left(h_{+1, a^{*}}\left(\min \left\{S_{u}^{*} \mid u \in\left[t, T_{1}\right]\right\}, V T\right) \geq 0\right)=0, \\
& Q^{*}\left(h_{-1, a^{*}}\left(\min \left\{S_{u}^{*} \mid u \in\left[t, T_{1}\right]\right\}, V T\right) \geq 0\right)=1 .
\end{aligned}
$$

If $S_{t}^{*}>\left(1+a^{*}\right) V T$, then

$$
\begin{aligned}
& Q^{*}\left(h_{+1, a^{*}}\left(\min \left\{S_{u}^{*} \mid u \in\left[t, T_{1}\right]\right\}, V T\right) \geq 0\right. \\
& \quad=1-Q^{*}\left(h_{-1, a^{*}}\left(\min \left\{S_{u}^{*} \mid u \in\left[t, T_{1}\right]\right\}, V T\right)>0\right),
\end{aligned}
$$

and we have

$$
\begin{aligned}
Q^{*} & \left(h_{+1, a^{*}}\left(\min \left\{S_{u}^{*} \mid u \in\left[t, T_{1}\right]\right\}, V T\right) \geq 0\right) \\
& =\frac{1}{2} \operatorname{Erfc}\left(\frac{A_{t}^{*}-(T-t) \mu^{*}}{\sigma^{*} \sqrt{2(T-t)}}\right)-\frac{1}{2} \exp \left(\frac{2 \mu^{*} A_{t}^{*}}{\left(\sigma^{*}\right)^{2}}\right) \operatorname{Erfc}\left(-\frac{A_{t}^{*}+(T-t) \mu^{*}}{\sigma^{*} \sqrt{2(T-t)}}\right) .
\end{aligned}
$$

4. Setting new targets. The previous section determined how the firm triggers disclosure by reference to a fixed decision rule $h$ and an observation process $X$ in terms of $V^{*}$, the proxy for market sentiment; above we took $X$ as being identical to this proxy for an observer-system of control theory, setting $X=V^{*}$. This replaces and simplifies the dynamics of the equilibrium approach of [GieO], but comes at the cost of losing information about the expected terminal firm-value (value at the next mandatory disclosure date). The model of GieO identifies that expected 'terminal value' as equal to the disclosed value. The modelling in the current section adapts and extends this line of reasoning into a construction of the target value VT introduced in Section 3. This 'plugs the gap between the models' by using an indifference level (see equations (1.1.1) in the Introduction).

If the firm were to use a threshold $L$ to trigger disclosure at some, for the moment arbitrary, future time moment $u$ in $(t, T]$, the firm's adopted decision rule, $h_{V T, u}$ say, determines disclosure iff $h_{V T, u}\left(u, X_{u}, L\right) \geq 0$. As only truthful disclosures are assumed, this entails a market valuation at whatever is the disclosed level. However, absence of a disclosure entails, for some appropriately selected threshold $L$, as in the model of [GieO], a valuation of $L$. In summary, if $L=L_{u}$ is selected appropriately for time $u$, then the time $u$ valuation of the firm is given by the random variable

$$
Z_{V T, u}\left(L_{u}\right)=X_{u} \mathbf{1}_{\left\{h_{V T, u}\left(u, X_{u}, L_{u}\right) \geq 0\right\}}+L_{u} \mathbf{1}_{\left\{h_{V T, u}\left(u, X_{u}, L_{u}\right)<0\right\}} .
$$


Now let $\tau_{V T}$ be a random time, with the interpretation that the event $\tau_{V T}(u)=u$ for $u>0$ means that $F$ observes $X_{u}$, the complementary event being $\tau_{V T}(u)=0$.

We now modify the random variables in (4.0.1a) by taking into account the times of observation and non-observation and define

$$
Z_{V T, u}^{\tau_{V T}}(L)=Z_{V T, u}(L) \mathbf{1}_{\left\{\tau_{V T}(u)=u\right\}}+L \mathbf{1}_{\left\{\tau_{V T}(u)=0\right\}},
$$

as a function of the level $L$. Then the expected valuation is

$$
\int_{(t, T]} E^{Q_{V T}}\left[Z_{V T, u}^{\tau_{V T}}\left(L_{u}\right) \mid \mathcal{F}_{t}\right] \tau_{V T}(\mathrm{~d} u),
$$

denoting here the distribution of $\tau_{V T}$ by $\tau_{V T}$ again, for notational convenience. As in Section 1.1 (cf. GieO]), since $Q_{V T}$ is risk-neutral, this should agree with $X_{t}$. Without loss of generality to the analysis of the interval $(t, T]$, we may agree to resize (rescale) the observation process at time $t$ to unity. Interpreting the values as discounted to present time $t$, our modelling assumption is to seek a constant $L=L_{V T}$ that solves

$$
1=\int_{[t, T]} E^{Q_{V T}}\left[Z_{V T, u}^{\tau_{V T}}\left(L_{V T}\right) \mid \mathcal{F}_{t}\right] \tau_{V T}(\mathrm{~d} u) .
$$

In setting the new target level, via the definition $V T=L_{V T}$, this formula relies not on the market filtration $\mathbf{F}^{*}$ (so not on future market sentiment), but on fair value computed from the larger filtration $\mathbf{F}$ with which the firm is equipped.

Granted the existence of a solution to (4.0.2), a matter addressed in Section 4.2 below, we take $L_{u}=L_{V T}=V T$ to correspond to each $h_{V T, u}$.

4.1. A benchmark observation scheme. For a tractable implementation of the modelling assumption in formula (4.0.2), we replace the random observation scheme $\tau$ by a deterministic one, known only to the firm but most certainly not known to the market. This permits a decomposition

$$
[t, T]=\mathcal{C}_{V T} \cup \mathcal{D}_{V T} \cup \mathcal{N}_{V T}
$$

according as observation extends over continuous intervals, or either at a finite number of (discrete) time moments, or not at all.

Then equation (4.0.2) reduces to finding some real $L$ such that we have

$$
\begin{aligned}
1 & =\int_{\mathcal{C}_{V T}} E^{Q_{V T}}\left[Z_{V T, u}^{\tau_{V T}}(L) \mid \mathcal{F}_{t}\right] \frac{\mathrm{d} u}{T-t} \\
& +\sum_{u \in \mathcal{D}_{V T}} E^{Q_{V T}}\left[Z_{V T, u}^{\tau_{V T}}(L) \mid \mathcal{F}_{t}\right] q_{V T}+\left(1-\frac{\operatorname{vol}\left(\mathcal{C}_{V T}\right)}{T-t}\right) L
\end{aligned}
$$

with $q_{V T}=1 / \# \mathcal{D}_{V T}$, effectively the constant probability of discrete monitoring.

Furthermore, taking $h_{V T, u}$ to be $h_{\varepsilon, a_{V T, u}}$ (with a mark-up $a_{V T, u}>-1$ ) leads to a decomposition of the variable $Z_{V T, u}$ into an option part and a non-option part, appropriately corresponding to good-news and bad-news events. For the good-news case $(\varepsilon=+1)$, it can readily be checked that this is

$$
Z_{V T, u}(L)=L+\max \left\{X_{u}-\left(1+a_{V T, u}\right) L, 0\right\}+a_{V T, u} L \mathbf{1}_{\left\{X_{u} \geq\left(1+a_{V T, u}\right) L\right\}},
$$

and similarly for bad-news $(\varepsilon=-1)$

$$
Z_{V T, u}(L)=L-\max \left\{\left(1+a_{V T, u}\right) L-X_{u}, 0\right\}+a_{V T, u} L \mathbf{1}_{\left\{X_{u} \leq\left(1+a_{V T, u}\right) L\right\}} .
$$


So the 'optionality' in $Z_{V T, u}(L)$ reduces to a plain vanilla option corrected by a digital option. Turning to the practicalities of options, one way to handle positions in digital options is to approximate them by plain vanilla positions using a selection of slightly amended strikes. From this perspective, the optionality of $Z_{V T, u}(L)$ can be regarded as approximately induced by a plain vanilla call- (respectively put-) option with strikes close to $\left(1+a_{V T, u}\right) L$. In view of its broader role we refer to $L_{V T}$ as the optimal censor (cf. Section 1.1).

Proposition 4.1 (Optimal censor optionality). When $a_{V T, u}=0$ for all $u$, with the additional assumption of only discrete observations $\left(\mathcal{C}_{V T}=\emptyset\right)$, the equation (4.1.2) for the optimal censoring thresholds specializes for the good-news event to

$$
1=2 L_{V T}+q_{V T} \sum_{u \in \mathcal{D}_{V T}} E^{Q_{V T}}\left[\max \left\{X_{u}-L_{V T}, 0\right\} \mid \mathcal{F}_{t}\right]
$$

and for bad-news

$$
1=2 L_{V T}-q_{V T} \sum_{u \in \mathcal{D}_{V T}} E^{Q_{V T}}\left[\max \left\{L_{V T}-X_{u}, 0\right\} \mid \mathcal{F}_{t}\right] .
$$

4.2. Existence of $L_{V T}$ for the benchmark observation scheme. This section demonstrates the existence of a target value $V T=L_{V T}$ for the benchmark observation scheme of the preceding subsection as characterized by equation (4.1.2). The existence theorems splits into two cases according as the decision rule determines good- or bad-news announcements; in both cases we analyze the functional form on the right of equation (4.1.2), treating $L$ as a free (non-negative real) variable. It is convenient to begin with bad-news announcements.

4.2.1. Bad-news case. The final term in equation (4.1.2), corresponding to non-monitoring, has a simple functional form: it is linear in $L$. To understand the other contributions, we rewrite the equation in a form which reflects the complementary conditioning in the two summands of the earlier equation (4.0.1b). This gives rise below to two corresponding functions of $L$ and recasts the characterization of $L_{V T}$ in the form:

$$
1=\mathcal{N}_{V T}\left(L_{V T}\right)+\mathcal{B S}_{V T, 1}\left(L_{V T}\right)+\mathcal{B S}_{V T, 2}\left(L_{V T}\right) .
$$

The three functions appearing here are defined as follows:

$$
\begin{aligned}
& \mathcal{N}_{V T}(L)=\left(1-\frac{\operatorname{vol}\left(\mathcal{C}_{V T}\right)}{T-t}\right) L, \\
& \mathcal{B S}_{V T, 1}(L)=\int_{\mathcal{C}_{V T}} E^{Q_{V T}}\left[X_{u} \mathbf{1}_{\left\{X_{u} \leq\left(1+a_{V T, u}\right) L\right\}} \mid \mathcal{F}_{t}\right] \frac{\mathrm{d} u}{T-t} \\
& +q_{V T} \sum_{u \in \mathcal{D}_{V T}} E^{Q_{V T}}\left[X_{u} \mathbf{1}_{\left\{X_{u} \leq\left(1+a_{V T, u}\right) L\right\}} \mid \mathcal{F}_{t}\right], \\
& \mathcal{B S}_{V T, 2}(L) / L=\int_{\mathcal{C}_{V T}} E^{Q_{V T}}\left[\mathbf{1}_{\left\{X_{u}>\left(1+a_{V T, u}\right) L\right\}} \mid \mathcal{F}_{t}\right] \frac{\mathrm{d} u}{T-t} \\
& +q_{V T} \sum_{u \in \mathcal{D}_{V T}} E^{Q_{V T}}\left[\mathbf{1}_{\left\{X_{u}>\left(1+a_{V T, u}\right) L\right\}} \mid \mathcal{F}_{t}\right]
\end{aligned}
$$

(with ' $\mathcal{B}$ for bad news' and ' $\mathcal{S}$ for Black-Scholes'). The relation between their behaviour and consequent existence of a target value is captured in the following result. 
Proposition 4.2. In bad-news events, for a constant $L_{V T}$ to exist for which equation (4.2.1a) holds, the following conditions (1) to (4) are sufficient.

(1) $\mathcal{B S}_{V T, 1}(L)$ and $\mathcal{B S}_{V T, 2}$ are continuous maps on $[0, \infty)$.

(2) $\mathcal{B S}_{V T, 1}(\infty)>-\infty$.

(3) $\operatorname{vol}\left(\mathcal{C}_{V T}\right) \neq T-t$, or $\mathcal{B S}_{V T, 2}$ is unbounded.

(4) $1 \geq \mathcal{B S}_{V T, 1}(0)$.

Proof. First consider the behaviour of the function summands as $L$ grows large. In $\mathcal{B S}_{V T, 1}(L)$ the indicator-functions for large $L$ will become those of the entire space, i.e. the constant function 1 ; the summands of $\mathcal{B S}_{V T, 1}(L)$ should thereby become expressible in terms of the first moments of $X$ as follows:

$(4.2 .1 \mathrm{c})_{\infty} \quad \mathcal{B S}_{V T, 1}(L)(\infty)=\int_{\mathcal{C}_{V T}} E^{Q_{V T}}\left[X_{u} \mid \mathcal{F}_{t}\right] \frac{\mathrm{d} u}{T-t}+q_{V T} \sum_{u \in \mathcal{D}_{V T}} E^{Q_{V T}}\left[X_{u} \mid \mathcal{F}_{t}\right]$.

Little can be said about the behaviour for large $L$ of $\mathcal{B S}_{V T, 2}(L)$ except for its being non-negative for $L$ non-negative. As a consequence,

$$
\mathcal{N}_{V T}(L)+\mathcal{B S}_{V T, 1}(L)+\mathcal{B S}_{V T, 2}(L) \geq \mathcal{N}_{V T}(L)+\mathcal{B S}_{V T, 1}(L),
$$

for any $L \geq 0$. On inspection from $(4.2 .1 \mathrm{~b})$, the right hand side of this inequality will grow linearly in $L$ arbitrarily provided $\operatorname{vol}\left(\mathcal{C}_{V T}\right) /(T-t) \neq 1$. Situations where $\operatorname{vol}\left(\mathcal{C}_{V T}\right)=T-t$ amount to monitoring $X$ at all points in time in $[t, T]$ except perhaps on an infinite sequence of points; this is contrary to the spirit of this paper's observation schemes $\tau_{V T}$, and so little will be lost in excluding such schemes. A minor problem arises, when $\mathcal{B S}_{V T, 1}(\infty)=-\infty$. Also, granting this technicality, the above line of reasoning gives conditions of unboundedness to the right (one is able to make the right-hand side of the inequality (4.2.1a) bigger than any given real by choosing $L$ sufficiently large); in particular, in the same way, it gives conditions for making the right-hand side bigger than 1.

Assume the functions are continuous in $L$. An application of the intermediate-value theorem will then establish the existence of $L_{V T}$ provided there is a value of $L$ for which the right-hand side of (4.2.1a) is smaller than 1 . There may be no way other than to postulate this, and it is most sensible to do so for the smallest value $L$ can take, namely 0.

4.2.2. Good-news case. We proceed similarly in this case, rewriting the characterizing equation again so as to reflect the relevant conditioning in (4.0.1). The difference here is that now a reversal of inequalities in the passage from bad- to good-news decisions requires corresponding new function definitions (below). These, alongside the term $\mathcal{N}_{V T}(L)$ from (4.2.1b), recast the existence problem to solving for $L_{V T}$ the equation

$$
1=\mathcal{N}_{V T}\left(L_{V T}\right)+\mathcal{G} \mathcal{S}_{V T, 1}\left(L_{V T}\right)+\mathcal{G} \mathcal{S}_{V T, 2}\left(L_{V T}\right) .
$$

Here (with ' $\mathcal{G}$ for good news') we define:

$$
\begin{aligned}
\mathcal{G S}_{V T, 1}(L) & =\int_{\mathcal{C}_{V T}} E^{Q_{V T}}\left[X_{u} \mathbf{1}_{\left\{X_{u} \geq\left(1+a_{V T, u}\right) L\right\}} \mid \mathcal{F}_{t}\right] \frac{\mathrm{d} u}{T-t} \\
& +q_{V T} \sum_{u \in \mathcal{D}_{V T}} E^{Q_{V T}}\left[X_{u} \mathbf{1}_{\left\{X_{u} \geq\left(1+a_{V T, u}\right) L\right\}} \mid \mathcal{F}_{t}\right],
\end{aligned}
$$




$$
\begin{aligned}
\mathcal{G S}_{V T, 2}(L) / L & =\int_{\mathcal{C}_{V T}} E^{Q_{V T}}\left[\mathbf{1}_{\left\{X_{u}<\left(1+a_{V T, u}\right) L\right\}} \mid \mathcal{F}_{t}\right] \frac{d u}{T-t} \\
& +q_{V T} \sum_{u \in \mathcal{D}_{V T}} E^{Q_{V T}}\left[\mathbf{1}_{\left\{X_{u}<\left(1+a_{V T, u}\right) L\right\}} \mid \mathcal{F}_{t}\right]
\end{aligned}
$$

Their behaviour and consequent relation to the existence of a target value is again captured by a result analogous to Proposition 4.2.

Proposition 4.3. In good-news events, for a constant $L_{V T}$ to exist for which equation (4.2.2a) holds, the two conditions (i) and (ii) below are sufficient.

(i) $\mathcal{G S}_{V T, 1}$ and $\mathcal{G S}_{V T, 2}$ are continuous maps on $[0, \infty)$.

(ii) $1 \geq \mathcal{G S}_{V T, 1}(0)$.

Proof. Mutatis mutandis, the line of reasoning developed for Proposition 4.2 now applies. Here, the larger $L$ is, the closer the indicator functions in $\mathcal{G S}_{V T, 2}(L)$ will come to the indicator function of the entire space; this translates into $\mathcal{G S}_{V T, 2}(L)$ becoming similar to some real $\mathcal{G S}_{V T, 2}(\infty)$ as $L$ grows large, and this real is positive. Since $\mathcal{G S}_{V T, 1}(L) \geq 0$ for every $L \geq 0$, the line of reasoning of Section 4.2.1 now establishes without further conditions the unboundedness in $L$ of the right-hand side of (4.2.2a).

4.2.3. Worked example in the geometric Brownian case. Corresponding to the mark-up decision rules of (2.0.1) there are six expectations appearing in the formulas of Sections 4.2.1 and 4.2.2 that are needed for an explicit determination of $L_{V T}$. Assume that $X$ follows geometric Brownian motion:

$$
X_{t+s}=X_{t} \exp \left(\left(\mu_{V T}-\frac{1}{2} \sigma_{V T}^{2}\right) s+\sigma_{V T} W_{V T, s}\right), \quad s \in[0, \infty),
$$

with $\mu_{V T} \in \mathbb{R}, \sigma_{V T}>0$, and $W_{V T}$ a standard Brownian motion independent of time $t$ information $\mathcal{F}_{t}$ as in (3.2.1). For fixed $u=t+s$ in $\left[t, T_{1}\right]$, these six expectations are provided by standard results on Brownian motion. Corresponding to $(4.2 .1 \mathrm{c})_{\infty}$ one has

$$
E^{Q_{V T}}\left[X_{u} \mid \mathcal{F}_{t}\right]=X_{t} \exp \left(\mu_{V T} s\right) ;
$$

likewise, corresponding to the pair (4.2.1d), (4.2.2c) and the pair (4.2.1c), (4.2.2b), taking

$$
\Delta_{s}:=\frac{\log \left(\left(1+a_{V T}\right) L / X_{t}\right)-\left(\mu_{V T}-\frac{1}{2} \sigma_{V T}^{2}\right) s}{\sigma_{V T}},
$$

one has respectively:

$$
\begin{aligned}
E^{Q_{V T}}\left[\mathbf{1}_{\left\{X_{u} \geq\left(1+a_{V T}\right) L\right\}} \mid \mathcal{F}_{t}\right] & =\frac{1}{2} \operatorname{Erfc}\left(\Delta_{s} / \sqrt{2 s}\right), \\
E^{Q_{V T}}\left[\mathbf{1}_{\left\{X_{u} \leq\left(1+a_{V T}\right) L\right\}} \mid \mathcal{F}_{t}\right] & =\frac{1}{2} \operatorname{Erfc}\left(-\Delta_{s} / \sqrt{2 s}\right), \\
E^{Q_{V T}}\left[X_{u} \mathbf{1}_{\left\{X_{u} \geq\left(1+a_{V T}\right) L\right\}} \mid \mathcal{F}_{t}\right] & =\frac{1}{2} X_{t} \exp \left(\mu_{V T} s\right) \operatorname{Erfc}\left(\frac{\Delta_{s}-\sigma_{V T} s}{\sqrt{2 s}}\right), \\
E^{Q_{V T}}\left[X_{u} \mathbf{1}_{\left\{X_{u} \leq\left(1+a_{V T}\right) L\right\}} \mid \mathcal{F}_{t}\right] & =\frac{1}{2} X_{t} \exp \left(\mu_{V T} s\right) \operatorname{Erfc}\left(-\frac{\Delta_{s}-\sigma_{V T} s}{\sqrt{2 s}}\right) .
\end{aligned}
$$

Here Erfc is again the complementary error function, for which specifically see Appendix equations (A.7ab) and (A.8). 
For periods of continuous monitoring, integrals of these three expressions need to be computed over time $s$. This is unproblematic for (4.2.3), where it reduces to differencing of the right-hand side across the endpoints of the monitoring period (and division by $\left.\mu_{V T}\right)$. For $(4.2 .5 \mathrm{ab})$ and $(4.2 .6 \mathrm{ab})$ this will lead to expressions in terms of non-standard special functions: the incomplete Bessel functions, given by integrals of the form $\int_{[t, T]} x^{\alpha} \exp \left(-\left(A / x^{2}+B^{2} x\right)\right) \mathrm{d} x$ for some real constants $A, B \geq 0$ and $\alpha$. Series representations can be derived for these integrals; generically in $\alpha$, the series are in terms of values of the incomplete gamma function, namely

$$
\begin{aligned}
& \int_{[t, T]} x^{\alpha} \exp \left(-\left(A / x^{2}+B^{2} x\right)\right) d x \\
= & \left(B^{2}\right)^{\alpha+1} \sum_{m=0}^{\infty} \frac{(-1)^{m}}{m !}\left(B^{2}\right)^{2 m}\left\{\Gamma\left(-(\alpha+m+1), \frac{B}{T}\right)-\Gamma\left(-(\alpha+m+1), \frac{B}{t}\right)\right\},
\end{aligned}
$$

where the series may be expressed in terms of Erfc only for particular choices of $\alpha$ (integer or half-integer values).

5. Comparative statics of early disclosure. Here we address matters on which GieO is silent.

\subsection{General performance index $\Sigma$}

THEOREM 5.1. With the modelling assumptions of Section 3.1, the following assertions hold in the framework of Sections 4.1 and 4.2 .

(1) Time-T disclosure becomes the more likely the smaller are $a^{*}$ and VT.

(2) In good-news situations time-T disclosure is the more likely the smaller is $r$ and the bigger are $a$ and $E_{T}^{*}$.

(3) In bad-news situations, time-T disclosure is the more likely the bigger is $r$ and the smaller are $a$ and $E_{T}^{*}$.

Proof. Working in the general process situation of Sections 4.1 and 4.2, the starting point here is the relevant set of inequalities holding at any time $T$ in $\left(T_{0}, T_{1}\right]$ which trigger a disclosure. In a good-news event this is

$$
V_{T} \geq(1+a) e^{-r\left(T_{1}-T\right)} E_{T}^{*} Q^{*}\left(\Sigma_{T, T_{1}}^{*} \geq\left(1+a^{*}\right) V T \mid \mathcal{F}_{T}^{*}\right) ;
$$

and for bad-news this is

$$
V_{T} \leq(1+a) e^{-r\left(T_{1}-T\right)} E_{T}^{*}\left(1-Q^{*}\left(\Sigma_{T, T_{1}}^{*}>\left(1+a^{*}\right) V T \mid \mathcal{F}_{T}^{*}\right)\right) .
$$

Treating $V T$ as a given (computed by the accounts department), the likelihood of the validity of these inequalities is determined by the size of the respective right-hand side; the good-news event (5.1.1) occurring is the more likely the bigger is the size of the expression on the right; the bad-news event (5.1.2) becomes the more likely the smaller is the size of the right. The variables on which the validity of these inequalities depend are thus: $a$ and $V T$, correspondingly $a^{*}$ and $E_{T}^{*}$, the interest-rate $r$, the 'time to maturity' $T_{1}-T$ (time left to the mandatory disclosure), and the variables beyond these that enter into the construction of $\Sigma_{T, T_{1}}^{*}$; the latter variables include $V C$ via $S_{T}^{*}$. 
REMARK 5.2. The effects of $S_{T}^{*}$ and $T_{1}-T$ on a time- $T$ disclosure decision depend on the specific form of the law of $\Sigma_{T, T_{1}}^{*}$. To justify this assertion as supplementary to Theorem 5.1, we look at the effects of infinitesimal changes in $S_{T}^{*}$ and $T_{1}-T$ on the (right-hand sides of) (5.1.1) and (5.1.2). Granted for simplicity the partial differentiability of these probabilities, we have the following two equations in terms of $\lambda_{T}^{*}$, the law of $\Sigma_{T, T_{1}}^{*}$ contingent on time- $T$ information $\mathcal{F}_{T}^{*}$ :

$$
\begin{aligned}
\partial_{T_{1}-T}\left[e^{r\left(T-T_{1}\right)} Q^{*}\left(\Sigma_{T, T_{1}}^{*}>\left(1+a^{*}\right) V T \mid \mathcal{F}_{T}^{*}\right)\right] \\
=e^{r\left(T-T_{1}\right)} \int_{\left(1+a^{*}\right) V T}^{\infty}\left\{\partial_{T_{1}-T} \lambda_{T}^{*}-r \lambda_{T}^{*}\right\}(u) \mathrm{d} u,
\end{aligned}
$$

$$
\partial_{S_{T}^{*}}\left[e^{r\left(T-T_{1}\right)} Q^{*}\left(\Sigma_{T, T_{1}}^{*}>\left(1+a^{*}\right) V T \mid \mathcal{F}_{T}^{*}\right)\right]=e^{r\left(T-T_{1}\right)} \int_{\left(1+a^{*}\right) V T}^{\infty} \partial_{S_{T}^{*}} \lambda_{T}^{*}(u) \mathrm{d} u .
$$

We see from these two equations that the signs of the effects depend on the exact form of this law, and so need to be determined on a case by case basis. Suffice it to say that conditions needing to be imposed here in concrete cases of $\lambda_{T}^{*}$ include conditions that entail that no sign changes occur in the respective integrands on the right-hand sides of these equations. To determine these signs explicitly requires concrete choices, at the least for $S^{*}$ and for how exactly $S^{*}$ enters into the definition of $\Sigma_{T, T_{1}}^{*}$.

5.2. Comparative statics for changes in $S_{T}^{*}$. We show that the assumption that the process $S^{*}$ follows a Markov process is sufficient for the determination of the effect of $S_{T}^{*}$ on early disclosure. So we work with processes with two properties: firstly that, for any time $u \geq 0$,

$$
S_{T_{0}+u}^{*}=S_{T_{0}}^{*} \exp \left(X_{u}^{*}\right), \quad \text { where } S_{T_{0}}=V C,
$$

where $\left(X_{u}^{*}\right)_{u \geq 0}$ is a process independent of time- $T_{0}$ information $\mathcal{F}_{T_{0}}^{*}$; secondly that, also for arbitrary $T \in\left(T_{0}, T_{1}\right]$,

$$
S_{T+u}^{*}=S_{T}^{*} \exp \left(X_{u}^{*}\right), \quad u \in[0, \infty),
$$

where, by abuse of language, $\left(X_{u}^{*}\right)_{u \geq 0}$ (or a suitable version of the process in (5.2.1) denoted by the same symbol) is a process independent of time- $T$ information $\mathcal{F}_{T}^{*}$.

THEOREM 5.3. We have $\partial_{S_{T}^{*}} Q^{*}\left(\Sigma_{T, T_{1}}^{*}>\left(1+a^{*}\right) V T \mid \mathcal{F}_{T}^{*}\right)>0$ in (5.1.4) under the additional Markovian conditions (5.2.1) and (5.2.2) on $S^{*}$; so in both good-news and bad-news situations a time-T disclosure becomes the more likely the larger is $S_{T}^{*}$. This conclusion holds more generally for all constructions of $\Sigma_{T, T_{1}}^{*}$ that preserve scaling (in the sense that we have $\left.\Sigma_{T, T_{1}}^{*}\left(S^{*}\right)=S_{T}^{*} \Sigma_{T, T_{1}}^{*}\left(\exp \left(X^{*}\right)\right)\right)$.

Proof. The assumptions (5.2.1) and (5.2.2) imply that $S^{*}$ is the product of a scaling factor $S_{T}^{*}$ and a 'standardized version' of $S^{*}$. This yields a representation for $\Sigma_{T, T_{1}}^{*}\left(S^{*}\right)$ as a product of two positive factors, the scaling factor $S_{T}^{*}$ and the random variable that results from the application of the respective construction for $\Sigma_{T, T_{1}}^{*}$ to the standardized version of $S^{*}$.

In (5.1.1) and (5.1.2) the partial derivatives with respect to $S_{T}^{*}$ of the probability factors will thus be positive so long as we have independence of the normalized versions of $S^{*}$ from time $T$-information. 
5.3. Early disclosure for geometric Brownian performance indices. We consider here the other parameter mentioned in Remark 5.2 , namely $T_{1}-T$, i.e. the time left to the next mandatory reporting date. At first sight, one might expect a proposition asserting that the shorter is this time, the less likely are decisions made for an early disclosure. However, on reflection, such decisions may well depend on the actual evolution of the market capitalization of the firm, and market forces may lead to changes in the size of this capitalization forcing early disclosure also at dates comparatively close to the mandatory date. Therefore, a discussion of the effects of $T_{1}-T$ needs to be incorporated in a model framework that includes $S^{*}$.

Here we adopt a standard Black-Scholes modelling for $S^{*}$, and therefore consider now the process $X^{*}$ in Section 5.2 as following scaled Brownian motion with drift:

$$
X_{u}^{*}=X^{*}\left(\mu^{*}, \sigma^{*}\right)_{u}=\mu^{*} u+\sigma^{*} W_{u}^{*}, \quad u \in[0, \infty),
$$

where $W^{*}$ is a $Q^{*}$-Brownian motion independent of time- $T$ information started with value 0 at time 0 , with parameters $\sigma^{*}>0$ and $\mu^{*}=r-\Delta^{*}-(1 / 2)\left(\sigma^{*}\right)^{2} \in \mathbb{R}$. Recall the former parameter is a measure of market volatility, while $r-\Delta^{*}$ is the excess of the (riskless) short rate, $r$, over the dividend rate of the firm $\Delta^{*}$ as seen by the markets; in the present context this difference should be viewed as an 'appreciation rate' for investments in $F$ (again as seen by the markets).

With $V^{*}$ modelled as in Proposition 3.1, the four effects to consider now are those induced by changes in $T_{1}-T$ and also in $\sigma^{*}, r-\Delta^{*}$, and $r$. These four will depend on which of good-news or bad-news situations occurs; they enter via the market proxies, and hence even a qualitative picture will depend on the concrete form of $\Sigma_{T, T_{1}}^{*}$. We focus on modelling $\Sigma_{T, T_{1}}^{*}$ as the running minimum or maximum of $S^{*}$, as in equations (3.1.5a) and $(3.1 .5 \mathrm{c})$, specifically in the good-news situations, so that by equation (5.1.1) we must consider the inequalities

$$
V_{T} \geq V_{\bullet}^{*}, \quad \bullet \in\{\max , \min \}
$$

with

$$
\begin{aligned}
V_{\max }^{*} & :=(1+a) E_{T}^{*} \exp \left(-r\left(T_{1}-T\right)\right) Q_{\max }^{*}, \\
Q_{\max }^{*} & :=Q^{*}\left(\max _{u \in\left[0, T_{1}-T\right]}\left\{X^{*}\left(\mu^{*}, \sigma^{*}\right)_{u}\right\}>A_{T}^{*}\right), \\
V_{\min }^{*} & :=(1+a) E_{T}^{*} \exp \left(-r\left(T_{1}-T\right)\right) Q_{\min }^{*}, \\
Q_{\min }^{*} & :=Q^{*}\left(\min _{u \in\left[0, T_{1}-T\right]}\left\{X^{*}\left(\mu^{*}, \sigma^{*}\right)_{u}\right\}>A_{T}^{*}\right),
\end{aligned}
$$

where

$$
A_{T}^{*}=\log \left(\left(1+a^{*}\right) V T / S_{T}^{*}\right) .
$$

5.3.1. Explicit results for geometric Brownian performance indices. We give a paradigm discussion of the effects of $T_{1}-T$ on the likelihood of disclosure decisions, when for the good-news case of (5.3.2) the running maximum is used in the construction of market proxies according to (5.3.3ab). These effects turn out to depend on the sign of the mark-up parameter $A_{T}^{*}$ as follows. 
THEOREM 5.4. In the framework of Section 5.3, assume a situation of time-T nondisclosure of good news. When using the running maximum of $S^{*}$ in Proposition 3.1 for $\Sigma_{T, T_{1}}^{*}$, the following two assertions are equivalent.

(1) Early disclosure in $\left[T, T_{1}\right]$ is the more likely the nearer is $T$ to $T_{1}$.

(2) $\operatorname{sign}\left(\partial_{T_{1}-T} V_{\max }^{*}\right)<0$.

Here the partial derivative in (2) depends on the sign of $A_{T}$; for $A_{T}^{*} \leq 0$ this is

$$
\partial_{T_{1}-T} V_{\max }^{*}=-r V_{\max }^{*},
$$

while for $A_{T}^{*} \geq 0$ this is

$$
\partial_{T_{1}-T} V_{\max }^{*}=-r V_{\max }^{*}+(1+a) E_{T}^{*} \exp \left(-r\left(T_{1}-T\right)\right) \frac{A_{T}^{*} \exp \left(-\eta^{2}\right)}{\sigma^{*} \sqrt{2 \pi\left(T_{1}-T\right)^{3}}},
$$

where

$$
\eta=\left(A_{T}^{*}-\left(T_{1}-T\right) \mu^{*}\right) / \sigma^{*} \sqrt{2\left(T_{1}-T\right)} .
$$

To indicate the typical line of reasoning for results like this, start from (5.3.2), observing that (in these good-news situations) disclosure decisions at some fixed point in time $T$ are the more likely the larger $V_{T}^{*}$ is. The effects of some parameter on the likelihood of early disclosure thus translate into the determination of the corresponding partial derivative of $V_{T}^{*}$, and so a determination of their qualitative effect reduces to a determination of the sign of these partials. Early disclosure thus becomes more likely the larger the relevant parameter, provided the corresponding partial of $V_{T}^{*}$ is positive, and vice versa. The point of our choice of a geometric Brownian framework is that explicit formulas for the probability factors $Q_{\bullet}^{*}$ are available as standard results in Brownian motion; these are reviewed in Appendix A below, with equations (A.7ab) pertinent for the present case of running-maximum performance parameters. Establishing comparative statics results therefore reduces to straightforward partial differentiation of explicitly given functions, albeit of some complexity. Theorem 5.3 collects the results when the relevant parameter there is the time left to the next mandatory date.

REMARK 5.5. Proceeding along the same lines in the same situation, one obtains results similar to those of Theorem 5.3 concerning the effects of the volatility $\sigma^{*}$, whereas the effects of $r-\Delta^{*}$ and $r$ are unequivocally unidirectional (with the signs of the pertinent partials being equal to minus that of $E_{T}^{*}$ ). Provided $E_{T}^{*}>0$, early disclosure within $\left(T, T_{1}\right)$ is the more likely the smaller are $r-\Delta^{*}$ and $r$.

5.3.2. Explicit results for running-min. Here we note only that if the market proxies are instead constructed using the running minimum of $S^{*}$ analogues of Theorem 5.3 and Remark 5.5 again hold and preserve all the conclusions above except for a sign reversal in $A_{T}^{*}$. This shows how derivation of the effects of $T_{1}-T$ on early disclosure requires the specifics of a given model.

6. Managerial implications. This paper's approach to asset pricing allows the development of a richer appreciation of how voluntary disclosure by firms can affect firm asset valuation in equilibrium. Existing research has typically modelled voluntary disclosure as the choice by firms to make additional voluntary disclosures to the market at fixed time 
points. As such this literature does not consider the possibility that firms may choose not only what to disclose voluntarily but also when to disclose. Thus voluntary disclosure has at least two dimensions: content and timing. As existing models typically do not consider the latter dimension, they are not truly dynamic, and hence do not provide the necessary building blocks to develop a realistic empirical model of ('two-dimensional') voluntary disclosure. Here we have explicitly modelled the joint content-timing interaction, so enabling more realistic formal modelling of problems faced by managers of firms: when private news is uncertain, how good does that private news have to be before it is in the interests of the firm to issue a voluntary disclosure. The other side of this coin is what materiality standard needs to be followed in managing the voluntary disclosure process. The comparative statics derived in the preceding section permit an understanding of how changes in parameter values may explain differences in equilibrium behaviour between firms - some voluntarily releasing additional information early, others not. This meets the challenge of modelling equilibrium asset-pricing with endogenously determined voluntary disclosures, wherein both the content and the timing of disclosures are rationally chosen, making delay or early release of information in capital markets an equilibrium outcome.

7. Complements. We close with some observations about the potential of the approach above especially with regard to variations on the themes presented and generalizations away from the Brownian framework followed above.

7.1. Mechanisms. Implicit in our development of a markets-based general modelling framework was the need to pick apart the 'who does what and how' into 'building bricks', and with these to build a variety of models. We implicitly identified five such bricks, which in fact are best considered as mechanisms, to borrow a phrase from economic theory. These are made explicit here so as to stress both the sensitivity of a model to its assumptions and its adaptability to alternative contexts.

Mechanism (i). Evolution rules. The perspective adopted above is rather like that of a scientist designing experiments and subsequently observing outcomes and evolution. Ingredients thus include design dynamics, start time and observation times. Thus mechanism (i) amounts to formal rules for encoding these three aspects. Real-life features mapped via such 'experiments' include informational interplay between economic agents and firm-to-market communications. See the summary in Section 7.5 for an explicit illustration of how this can be further developed.

Mechanism (ii). Decision-rule strategies. The task here is to provide rules for triggering 'events' (typically, public disclosure of privileged information), and the idea is that these be the consequence of some 'rule', i.e. functional relation, applied to some observation variables. At a technical level, this mechanism thus amounts to the selection of functional relations subject to the specification of observation variables. The mechanisms we adopted, starting from Section 3, are motivated by the provision of approximations to equilibrium-induced decision rules as derived in [GieO]. There they correspond to 'value-enhancement' disclosures when observations are 'sufficiently high'. Whilst outside the scope of the present paper, the argument there may be dualized to correspond to 
equilibrium-induced 'value-erosion' alerts when observations are 'sufficiently low', with a resulting notion of endogeneous 'materiality thresholds'. Such an understanding leads to the following

Conjecture 7.1. First-order approximation of equilibrium decision-rules based on the notion of materiality yields decision-rules using functions taking the form

$$
h_{\varepsilon, a}(t, x, y)=\left(x-\left(1+a_{t}\right) y\right) \varepsilon_{t}
$$

for some families of signs $\varepsilon=\left(\varepsilon_{t}\right)_{t \in\left[T_{0}, T_{1}\right]}$ and mark-ups $a=\left(a_{t}\right)_{t \in\left[T_{0}, T_{1}\right]}$, and vice versa.

Mechanism (iii). Observation processes. These generalize the processes $V$ introduced in Sections 1.1 and 2, and give expression to the fundamental notion of informational asymmetry. Here the task is to construct a 'variable' (which can be multi-dimensional) with two properties. Firstly, it is capable of observation over time and is observed over time by the informationally privileged agents of whatever model is to be constructed (denoted by the symbol $F$, typifying firms); secondly, the variable is at best partially observable by the remaining agents (denoted by the symbol $M$ ).

As to observation variables, we focus on a portfolio view. Continuing to think of $F$ as a firm for a moment, $F$ will not in general observe just a single source of information to set a target, but a portfolio of these, say $\left(X_{1}, \ldots, X_{n}, \ldots, X_{N}\right)$. Formally, the chosen observation process $X$ will be a function of the $X_{n}$; simple, but typical, functions are linear or multiplicative forms in the $X_{n}$ as given respectively by

$$
X=\sum_{n=1}^{N} a_{n} X_{n}, \text { or } X=\prod_{n=1}^{N} X_{n}^{a_{n}},
$$

with suitable real weights $a_{n} \in \mathbb{R}$.

Examples of two factor portfolio observation variables. In the paper, we considered the case where $X=V$ and $V$ represented the value of the firm $F$, i.e., a process internally observed by $F$ subject to privileged private information. It is natural to complement it by a process that encodes the external view of the firm's value subject to non-privileged public information, such as provided by the firm's market capitalization, $S^{*}$. Specializing to portfolios of additive type, the associated observation variable may take the form

$$
X=a V+b S^{*}
$$

for some $a, b \in \mathbb{R}$. General structure of $X$ apart, the modelling of $V$ and $S^{*}$ is far from straightforward, and Sections 7.2 to 7.4 below offer an amendment to the simplified treatment given in the main body of the paper.

Mechanism (iv). Observation process proxies $V^{*}$ from state observer systems. The task here is to enable specifically the 'informationally under-privileged' agents in the models to approximate $V$. To paraphrase a key idea in the paper: here $S^{*}$ is seen as a proxy for $V$ in creating an estimate $V_{t}^{*}$ of $V_{t}$ subject to public information; for tractability we made specific approximation assumptions.

In so doing, we borrowed an idea from the control theory of an engineering plant, where one way to deal with imperfect information about the plant is to build a laboratory version (a model) of the plant with accessible full-information of its state at any time (known as a 'state observer' system [Rus, Chapter 3], [Son, Chapter 7] — in reality a 'state 
estimator'); state-correcting signals are sent to this model, using plant-based, imperfect, or partial observations, with which to guide the 'observer system' (model) into greater agreement with the plant.

Unlike in the engineering context, inclusion into a market-based model of an observerstyle system implies changes to the strategic behaviour of the firm in its decisions to hide certain observations of its state. Indeed, here each side (each of the agents, $F$ or $M$ ) enriches its algorithmic opportunities. In this context, our version of an 'observer system' responds to strategic behaviour, so is far richer. To emphasize this difference when, for example, $F$ was a firm, the observer-style system was termed a proxy-firm.

Mechanism (v). Target value processes VT. Under this heading, the task is to provide techniques for forecasting values of observation variables (and, significantly, of their proxies), bearing in mind that such items are contingent on market developments as well as on restrictions arising from (production) technologies. In Section 4 we marry accounting analysis with the analysis of Bermudean options: mechanism (v) involved observation levels $L_{t}$ for $V_{t}$ qua strike prices at which the respective agent's decision rule is indifferent between disclosing or suppressing private observation of $V_{t}$, were $V_{t}$ to take the value $L_{t}$ (as noted in the Introduction). This construction borrows from the stylized model of [GieO], where these levels describe the market's current view of the value during periods of silence and so provide the basis of current guidance on its earnings target.

7.2. Modelling firm-value processes: uncertainty structure of profits. As to the observation mechanism, one may 'drill down' to the basic structure of profits and address the uncertainty effects created by reporting lags. The starting point is a formalization of accounting practice: $V_{u}$ the firm $F$ 's time- $u$ value is the accrual of an instantaneous variable $\pi_{w}$ over the period $\left[T_{0}, u\right]$ added to an initially given value $V C$ of time $T_{0}$, so that

$$
V_{u}=V C+\int_{\left[T_{0}, u\right]} \pi_{w} \mathrm{~d} w, \quad u \in\left[T_{0}, T_{1}\right],
$$

implicitly assuming $w \mapsto \pi_{w}$ to be summable over $\left[T_{0}, T_{1}\right]$.

The simplest interpretation of $V_{u}$ is incrementing $V C$ by the firm's actual profit flow rate $\pi_{w}$ - as it actually arises at each time moment $w$ between time- $T_{0}$ and time- $u$. Alternatively, to allow for the possibility of delays in reporting profits (due, say, to reporting delays of costs, as below), we can re-interpret this as the recognized profit flow rate - namely, the value posted in some official ledger.

To introduce reporting lags into the model, fix $\Lambda \geq 0$ and then at each time $u$, assume the flow $\pi_{w}$ is certain only for 'distant' times $w$, namely times earlier than $u-\Lambda$, but for times $w$ nearer to $u$, i.e. in $(u-\Lambda, u], \pi_{w}$ is uncertain. A further refinement then occurs in the decomposition (7.2.1) created by a deterministic part $\Delta^{\mathrm{ns}} V_{u}$, certain at time- $u$ (with 'ns' for non-stochastic), and a part $\Delta^{\mathrm{s}} V_{u}$ uncertain at time-u:

$$
V_{u}=V C+\Delta^{\mathrm{ns}} V_{u}+\Delta^{s} V_{u}, \quad u \in\left[T_{0}, T_{1}\right]
$$

where

$$
\Delta^{\mathrm{ns}} V_{u}=\int_{\left[T_{0}, \max \left\{T_{0}, u-\Lambda\right\}\right]} \pi_{w} \mathrm{~d} w,
$$




$$
\Delta^{\mathrm{s}} V_{u}=\int_{\left[\max \left\{T_{0}, u-\Lambda\right\}, u\right]} \pi_{w} \mathrm{~d} w .
$$

We view $V_{u}^{\mathrm{ns}}=V C+\Delta^{\mathrm{ns}} V_{u}$ as an accounting equality, namely, as data held, or stored, by the firm $F$, and $\Delta^{\mathrm{s}} V_{u}$ as a variable that needs to be modelled - e.g., as in Section 7.3 below.

Two obvious questions arise: first, how does (a manager) $F$ respond to such operational uncertainty. For example, will there be a period in which $F$ is waiting for the time- $T$ accounting information to be corroborated and to be verified as reliable (up to a level of security deemed appropriate for the decision-making), and how does $F$ then respond to the evolution of market sentiment during such periods of waiting? Second, is there a correlation between market sentiment $V^{*}$ and the degree of operational effectiveness of the firm's accounting department?

7.3. Modelling firm-value processes: Cobb-Douglas examples. As a second complement to our discussion of mechanism (iii), we provide examples for modelling firm-value observation processes $V$ concretely.

7.3.1. Deterministic Cobb-Douglas profits. Here the construction of $V$ needs to be linked to the standard functional forms preferred by the theory of the firm in Economics and Econometrics. We consider Cobb-Douglas technologies, and indicate how to model profits derived from a Cobb-Douglas production function ( $\mathrm{Var}$, Chapter 1, especially Examples 1.10, 1.11], [Rom, Chapter 2]) corresponding to a single output from two input factors (such as capital and labour) with respective parameters $a, b \geq 0$ satisfying $a+b<1$. This yields profits as a function of input prices $w=\left(w_{1}, w_{2}\right)$ and output selling-price $p$ in the form

$$
\begin{aligned}
& \pi_{C D}(p, w) \\
& =p^{1 /(1-(a+b))}\left\{\left(\frac{a+b}{\kappa \cdot c(w)}\right)^{(a+b) /(1-(a+b))}-\kappa \cdot c(w)\left(\frac{a+b}{\kappa \cdot c(w)}\right)^{1 /(1-(a+b))}\right\},
\end{aligned}
$$

for a cost function $c(w)=\left(w_{1}^{a} w_{2}^{b}\right)^{1 /(a+b)}$, where

$$
\kappa:=\left((a / b)^{a /(a+b)}+(a / b)^{-(a /(a+b)}\right) / A^{1 /(a+b)} \text {, with } A=(1-a)^{a-1} / a^{a} .
$$

7.3.2. Stochastic Cobb-Douglas profits. To take into account uncertainties in the profit function, assume that uncertainty in the output and input price is given by positive stochastic processes on some stochastic basis, say $\mathcal{X}(Q)=\left(\Omega, \mathcal{F}, \mathbf{F}=\left(\mathcal{F}_{u}\right)_{u \in\left[T_{0}, T_{1}\right]}, Q\right)$. As regards output, passing to logarithmic prices and so to an exponential price process $\exp Y,(7.2 .1)$ yields profits of the form

$$
\left.\pi_{w}=\alpha \exp \left(Y_{w}\right)^{\beta}=\exp \left(\log (\alpha)+\beta Y_{w}\right)\right), \quad w \in\left[T_{0}, T_{1}\right],
$$

for some fixed $\alpha>0, \beta \in \mathbb{R}$, and some fixed stochastic process $Y$ on $\mathcal{X}(Q)$. In turn this gives the uncertain part of the time- $u$ value of $V$ the representation:

$$
\Delta^{\mathrm{s}} V_{u}=\alpha \int_{\left[\max \left\{T_{0}, u-\Lambda\right\}, u\right]} \exp \left(\beta Y_{w}\right) \mathrm{d} w, \quad u \in\left[T_{0}, T_{1}\right] .
$$

Treating input (factor) prices $w$ in similar vein preserves this general form for the uncertain parts of $V$ in (7.2.2c). In any of these representations, a notable choice for $Y$ is 
Brownian motion on $\mathcal{X}(Q)$, and this provides an explicit illustration of how the modelling above turns $V_{u}$ itself into a random variable, given the time- $u$ information (see Section 7.4 for scalable processes, which we consider here as candidate modelling mechanisms).

As mentioned, accruals in (7.2.1) can be modelled in (at least) two conceptually different ways, depending on whether instantaneous profits $\pi_{s}$ or their accumulated value is taken as a primary variable. The matter of choice is not just a conceptual but also a practical one, even assuming the classical Cobb-Douglas two-factor production technology above. For this purpose re-write (7.3.1) in the form

$$
\begin{aligned}
& \text { (7.3.4a) } \pi_{C D}\left(p, w_{1}, w_{2}\right) \\
& =w_{1}^{a /(a+b-1)} w_{2}^{b /(a+b-1)} p^{(a+b-1)}\left\{\left(\frac{a+b}{\kappa}\right)^{(a+b) /(1-(a+b))}-\kappa\left(\frac{a+b}{\kappa}\right)^{1 /(1-(a+b))}\right\},
\end{aligned}
$$

with constants $a, b>0$ such that $a+b<1$ for

$$
\kappa:=\left((a / b)^{b /(a+b)}+(a / b)^{-a /(a+b)}\right) /\left((1-a)^{a-1} / a^{a}\right)^{1 /(a+b)} .
$$

Assume that non-deterministic profits are the result of fluctuations in any of these prices, and, for simplicity, staying within the Brownian framework, assume the fluctuations follow geometric Brownian motion. The resulting dynamic for $\pi_{\mathrm{CD}}$ is of the form

$$
\pi_{\mathrm{CD}, T+u}=\pi_{\mathrm{CD}, T} \exp \left(\mu_{\pi} u+\sigma_{\pi} W_{\pi, u}\right), \quad u \in[0, \infty),
$$

with driver a Brownian motion $W_{\pi}$ independent of the information available for forecasting at time $T$ (as provided via the filtration of a stochastic basis specified for forecasting purposes) and with real constants $\sigma_{\pi}>0$ and $\mu_{\pi}$. Return now to the choice of explicit modelling variants; according to the choice of accumulated profits or instantaneous profits, one has respectively

$$
\begin{gathered}
\pi_{s}=\pi_{C D, T+s}, \quad s \in[0, \infty), \\
\int_{[T, T+s]} \pi_{w} \mathrm{~d} w=\pi_{C D, T+s}, \quad s \in[0, \infty) .
\end{gathered}
$$

This last requires for $V_{T+u}$ the integral of geometric Brownian motion, not covered by the context of Section 4. For the first, the results of Section 4.2 do apply, and provide the forecast target $V T$.

7.4. Scalable processes. As a third complement to Section 7.1, we suggest the use scalable processes for modelling with mechanisms. These processes will be patterned after the exponentials of strong Markov processes $S^{*}$, which satisfy two equations. Firstly, with $T_{0}$ fixed, for any time $u \geq 0$,

$$
S_{T_{0}+u}^{*}=S_{T_{0}}^{*} \exp \left(X_{u}^{*}\right), \quad \text { where } \quad S_{T_{0}}^{*}=V C,
$$

where $\left(X_{u}^{*}\right)_{u \geq 0}$ is independent of time- $T_{0}$ information $\mathcal{F}_{T_{0}}^{*}$. Secondly, for arbitrary real $T$ in $\left(T_{0}, T_{1}\right]$, the representation

$$
S_{T+u}^{*}=S_{T}^{*} \exp \left(X_{u}^{*}\right), \quad u \in[0, \infty),
$$

where, by abuse of language, $\left(X_{u}^{*}\right)_{u \geq 0}$ (or a suitable version of the process in (7.4.1) denoted by the same symbol) is a process independent of time- $T$ information $\mathcal{F}_{T}^{*}$. We now relax the second condition and define processes $S^{*}$ to be scalable if they are RCLL and satisfy (7.4.1) and (7.4.2), except that now (7.4.2) need not necessarily hold for all $T$ 
in $\left(T_{0}, T_{1}\right]$ and instead is to hold necessarily for all $T$ in some prespecified finite subset $\mathcal{T}$ of $\left(T_{0}, T_{1}\right]$. Here we primarily think of $\mathcal{T}$ as containing the endpoints of benchmark observation schemes along the lines of equation (4.1.1). Extending this notion of a scalable process to allow the sets $\mathcal{T}$ to have at most countably many stopping times should not, however, pose problems.

7.5. Modelling with mechanisms: a summary vista. A characteristic feature of the mechanisms identified in Section 7.1 is that they identify the economic agents solely in terms of how they act. In respectively Sections 4 and 3, as it happens, the agents $F$ posited by the mechanisms in disclosure situations are indeed interpreted as acting like the manager of the firm, and agents $M$ as acting like representative market participants. For the wider guidance theme, however, specific market participants will also 'act out' the role of agent $F$ within some of the mechanisms. An outline follows.

To tell our guidance story we need to single out a distinguished group of people from among the market participants $M$, whom we shall call analysts. The typical representative member of this group being denoted by $A$, we continue to denote representative market participants as agents $M$ (as in Section 3).

The guidance theme then starts at time $t$ with the announcement by manager $F$ of the current accounting numbers of the firm: $V C$ and its target $V T$ for the next mandatory reporting date, $T$ below. These numbers are processed by $M$ as in the disclosure theme, while now $A$ is also assumed to make its own computations. For these computations $A$ will be assumed to use mechanism (v) of Section 7.1 (as though in the role of agent $F$ ), and make its own computations of the time- $T$ target, possibly based on a re-estimation of $V, V^{*}, S$ and $S^{*}$; call the result $V T_{A}$, and assume that $A$ will announce this number to $M$ and $F$ at a time $t+\Delta_{A}$ within $[t, T]$. This announcement will possibly induce a re-calibration of the price processes $S$ and $S^{*}$; the analyst's target $V T_{A}$ will now be added as a new variable in the decision making of the manager $F$. Apart from a possible consequent re-calibration of $V^{*}$, the essential difference from the set-up of Section 3 is that now manager $F$ is assumed to use decision rules in four variables, say $h\left(T, V_{T}, V_{T^{*}}, V T_{A}\right)$. For present outline purposes, we will not make this four-variable rule explicit, leaving the details to be established elsewhere. Now running the disclosure-mechanisms of Sections 3,4 , and 5 results, mutatis mutandis, in either an early disclosure at some time $\tau<T$, or a regular one at time $T$. In both cases an announcement is made by manager $F$ of new current numbers $V C$ and targets $V T$ for the next reporting date; these numbers will be announced simultaneously to the analysts $A$ and to the market (as represented by agent $M$ ) and the entire activity starts all over again. The details are intended to be established elsewhere.

Appendix: Reductions. This section collects the simplifications arising for general mark-up decision rules in Brownian contexts. We work with a fixed probability space $(\Omega, \mathcal{F}, P)$ which is equipped with a filtration $\mathbf{F}=\left(\mathcal{F}_{u}\right)_{u \geq 0}$ such that the resulting stochastic basis $\mathcal{X}(P)=(\Omega, \mathcal{F}, \mathbf{F}, P)$ satisfies the usual conditions (see for example [JacS Def. 1.3, p. 2]). The mark-up decision rules are assumed, as above, in the form $h_{\varepsilon, a}(x, y)=\varepsilon(x-(1+a) y)$, for fixed parameters $\varepsilon= \pm 1$ and $a>-1$. 
A.1. Bad-news to good-news reductions. For $\Sigma$ a random variable on $\Omega$, measurable with respect to $\mathcal{F}_{t}$, and fixed $t>0$,

$$
\begin{aligned}
& E^{P}\left[\mathbf{1}_{\left\{h_{+1, a}(\Sigma, V T) \geq 0\right\}} \mid \mathcal{F}_{t}\right]=P\left(\{\Sigma \geq(1+a) V T\} \mid \mathcal{F}_{t}\right), \\
& E^{P}\left[\mathbf{1}_{\left\{h_{-1, a}(\Sigma, V T) \geq 0\right\}} \mid \mathcal{F}_{t}\right]=1-P\left(\{\Sigma>(1+a) V T\} \mid \mathcal{F}_{t}\right) .
\end{aligned}
$$

These two yield a reduction of bad-news to good-news disclosures via

$$
E^{P}\left[\mathbf{1}_{\left\{h_{-1, a}(\Sigma, V T) \geq 0\right\}} \mid \mathcal{F}_{t}\right]=1-E^{P}\left[\mathbf{1}_{\left\{h_{+1, a}(\Sigma, V T) \geq 0\right\}} \mid \mathcal{F}_{t}\right],
$$

granted absence of point-masses in $\Sigma$ located at $(1+a) V T$, given the continuous processes in play here. Note the qualitative consequence that factors influencing the relevant probabilities will have opposite effects on good-news and bad-news events; an increase in a factor that leads to an increase of (A.0a) will decrease (A.1) and vice versa.

A.2. Running-minimum to running-maximum reductions. We collect here the further reductions needed for good-news events when $S^{*}$ is a geometric Brownian motion. With $\Sigma^{*}=\Sigma_{t, T_{1}}^{*}$ the running maximum or the running minimum of $S^{*}$ on some fixed time interval $\left[t, T_{1}\right]$, let $W^{*}$ be an $(\mathbf{F}, P)$-Brownian motion on $\mathcal{X}(P)$ started at 0 at time 0 ; for $\sigma^{*}>0$ take

$$
S_{t+u}^{*}=S_{t}^{*} \exp \left(\mu^{*} u+\sigma^{*} W_{u}^{*}\right), \quad u \in[0, \infty),
$$

with $\mu^{*}=r-\delta-\frac{1}{2}\left(\sigma^{*}\right)^{2}$ for $r-\delta \in \mathbb{R}$; appealing to the strong Markov property of Brownian motion, assume also $W^{*}$ to be independent of $\mathcal{F}_{t}$, and express the events in terms of $W^{*}$ as follows:

$$
\begin{aligned}
& E^{P}\left[\mathbf{1}_{\left\{h_{+1, a}\left(\max \left\{S_{w}^{*} \mid w \in[t, T]\right\}\right) \geq 0\right\}} \mid \mathcal{F}_{t}\right]=P\left(\max _{u \in[0, T-t]}\left\{\mu^{*} u+\sigma^{*} W_{u}^{*}\right\} \geq A_{t}^{*}\right), \\
& E^{P}\left[\mathbf{1}_{\left\{h_{-1, a}\left(\min \left\{S_{w}^{*} \mid w \in[t, T]\right\}\right) \geq 0\right\}} \mid \mathcal{F}_{t}\right]=P\left(\min _{u \in[0, T-t]}\left\{\mu^{*} u+\sigma^{*} W_{u}^{*}\right\} \leq A_{t}^{*}\right),
\end{aligned}
$$

with

$$
A_{T}^{*}:=\log \left(\left(1+a^{*}\right) V T / S_{t}^{*}\right) .
$$

Setting $W^{* *}:=-W^{*}$ note that

$$
P\left(\min _{u \in[0, T-t]}\left\{\mu^{*} u+\sigma^{*} W_{u}^{*}\right\} \leq A_{t}^{*}\right)=P\left(\max _{u \in[0, T-t]}\left\{-\mu^{*} u+\sigma^{*} W_{u}^{* *}\right\} \geq-A_{t}^{*}\right) .
$$

It is special to the Brownian context that (A.6) provides a reduction of the runningminimum event in (A.4) to a running-maximum event in (A.3), since, if $W^{*}$ is Brownian, then so is $W^{* *}$ as its negative. An explicit determination of the expectation (A.3) can be had from the explicit law for the running-maximum of Brownian motion; see e.g. [Fre, (30) Corollary, p. 25]. This relies, in this Brownian context, on the running-maximum always being positive on time intervals of positive length; indeed, this follows from the fact that the running-maximum of the process is zero on non-positive time arguments. This is not directly of use here, since the drift $\mu^{*}$ is in general non-zero. But an appropriate Girsanov transformation applied to the measure $P$ will achieve a reduction to the zerodrift case (cf. $[\operatorname{RogW} \S \mathrm{I}$ I.13, eq. (13.9)]), at the cost, however, of an additional exponential factor in (A.3); the results are as follows:

$$
P\left(\max _{u \in[0, T-t]}\left\{\mu^{*} u+\sigma^{*} W_{u}^{*}\right\} \geq A_{t}^{*}\right)=1,
$$


unless $A_{t}^{*}>0$, in which case

$$
\begin{aligned}
& P\left(\max _{u \in[0, T-t]}\left\{\mu^{*} u+\sigma^{*} W_{u}^{*}\right\} \geq A_{t}^{*}\right) \\
& \quad=\frac{1}{2} \operatorname{Erfc}\left(\frac{A_{t}^{*}-(T-t) \mu^{*}}{\sigma^{*} \sqrt{2(T-t)}}\right)+\frac{1}{2} \exp \left(\frac{2 \mu^{*} A_{t}^{*}}{\left(\sigma^{*}\right)^{2}}\right) \operatorname{Erfc}\left(\frac{A_{t}^{*}+(T-t) \mu^{*}}{\sigma^{*} \sqrt{2(T-t)}}\right),
\end{aligned}
$$

with $\operatorname{Erfc}(z):=(2 / \sqrt{\pi}) \int_{[z, \infty)} \exp \left(-w^{2}\right) d w$, for any complex $z$, the complementary error function. This result can be established, mutatis mutandis, along the lines of [MusR, Lemma A.18.2, p. 617seq]); for a proof by a reduction to this result, start from the equality

$$
P\left(\max _{u \in[0, T-t]}\left\{\mu^{*} u+\sigma^{*} W_{u}^{*}\right\} \geq A_{t}^{*}\right)=1-P\left(\max _{u \in[0, T-t]}\left\{\mu^{*} u+\sigma^{*} W_{u}^{*}\right\} \leq A_{t}^{*}\right) ;
$$

on the right-hand side we have from [MusR, eq. (A.85)] the equality

$$
\begin{aligned}
P\left(\max _{u \in[0, T-t]}\left\{\mu^{*} u+\sigma^{*} W_{u}^{*}\right\}\right. & \left.\leq A_{t}^{*}\right) \\
& =N\left(\frac{A_{t}^{*}-(T-t) \mu^{*}}{\sigma^{*} \sqrt{T-t}}\right)-\exp \left(2 \frac{\mu^{*} A_{t}^{*}}{\left(\sigma^{*}\right)^{2}}\right) N\left(-\frac{A_{t}^{*}+(T-t) \mu^{*}}{\sigma^{*} \sqrt{T-t}}\right),
\end{aligned}
$$

if $A_{t}^{*} \geq 0$, but otherwise this probability is 0 ; then successively use the identities $1=$ $N(\xi)+N(-\xi)$ and $N(\xi)=(1 / 2) \operatorname{Erfc}(-\xi / \sqrt{2})$ to arrive at (A.7ab).

Formulas for the tails of the running-minimum expressions of (A.4) are a consequence of (A.7ab). For this start by passing to the complementary probability

$$
P\left(\min _{u \in[0, T-t]}\left\{\mu^{*} u+\sigma^{*} W_{u}^{*}\right\} \geq A_{t}^{*}\right)=1-P\left(\min _{u \in[0, T-t]}\left\{\mu^{*} u+\sigma^{*} W_{u}^{*}\right\} \leq A_{t}^{*}\right) ;
$$

now use (A.6) to translate the right-hand side in terms of probabilities for the running maximum, and apply (A.7ab) to obtain

$$
\begin{aligned}
& P\left(\min _{u \in[0, T-t]}\left\{\mu^{*} u+\sigma^{*} W_{u}^{*}\right\} \geq A_{t}^{*}\right) \\
& \quad=\frac{1}{2} \operatorname{Erfc}\left(+\frac{A_{t}^{*}-(T-t) \mu^{*}}{\sigma^{*} \sqrt{2(T-t)}}\right)-\frac{1}{2} \exp \left(\frac{2 \mu^{*} A_{t}^{*}}{\left(\sigma^{*}\right)^{2}}\right) \operatorname{Erfc}\left(-\frac{A_{t}^{*}+(T-t) \mu^{*}}{\sigma^{*} \sqrt{2(T-t)}}\right),
\end{aligned}
$$

unless $A_{t}^{*} \geq 0$, in which case the probability is equal to 0 ; to obtain the first summand here use the identity $2=\operatorname{Erfc}(\xi)+\operatorname{Erfc}(-\xi)$ with

$$
\xi:=\left(A_{t}^{*}-(T-t) \mu^{*}\right) /\left(\sigma^{*} \sqrt{2(T-t)}\right) .
$$

Acknowledgments. We thank the Referee and the Editor for a close reading of the paper and advice which has led to presentational improvements.

\section{References}

[AtiLST] R. K. Atiase, H. Li, S. Supattarakul, S. Tse, Market reaction to multiple contemporaneous earnings signals: Earnings announcements and future earnings guidance, Rev. Accounting Stud. 10 (2005), 497-525.

[BaiD] S. Baiman, J. S. Demski, Economically optimal performance evaluation and control systems, J. Accounting Res. 18 (1980), 184-220. 
[BozRB] Z. Bozanic, D. T. Roulstone, A. Van Buskirk, Management earnings forecasts and other forward-looking statements, J. Accounting and Economics 65 (2018), 1-20.

[CheJY] M. Chesney, M. Jeanblanc-Picqué, M. Yor, Brownian excursions and Parisian barrier options, Adv. in Appl. Probab. 29 (1997), 165-184.

[Dye] R. A. Dye, Disclosure of nonproprietary information, J. Accounting Res. 23 (1985), $123-145$.

[EinZ] E. Einhorn, A. Ziv, Intertemporal dynamics of corporate voluntary disclosures, J. Accounting Res. 46 (2008), 567-589.

[Fre] D. Freedman, Brownian Motion and Diffusion, second ed., Springer, New York, 1983.

[GieO] M. B. Gietzmann, A. J. Ostaszewski, The sound of silence: equilibrium filtering and optimal censoring in financial markets, Adv. in Appl. Probab. 48 (2016), 119-144.

[HirKV] D. E. Hirst, L. Koonce, S. Venkataraman, Management earnings forecasts: A review and framework, Accounting Horizons 22 (2008), 315-338.

[JacS] J. Jacod, A. N. Shiryaev, Limit Theorems for Stochastic Processes, Grundlehren Math. Wiss. 288, Springer, Berlin, 1987.

[Jeu] T. Jeulin, Semi-martingales et grossissement d'une filtration, Lecture Notes in Math. 833, Springer, Berlin, 1980.

[JogM] V. Jog, B. J. McConomy, Voluntary disclosure of management earnings forecasts in IPO prospectuses, J. Business Finance \& Accounting 30 (2003), 125-168.

[JunK] W.-O. Jung, Y. K. Kwon, Disclosure when the market is unsure of information endowment of managers, J. Accounting Res. 26 (1988), 146-153.

[KocLR] A. S. Koch, C. E. Lefanowicz, J. R. Robinson, Regulation FD: A review and synthesis of the academic literature, Accounting Horizons 27 (2013), 619-646.

[MusR] M. Musiela, M. Rutkowski, Martingale methods in financial modelling, second ed., Stoch. Model. Appl. Probab. 36, Springer, Berlin, 2005.

[RogSB] J. L. Rogers, D. J. Skinner, A. Van Buskirk, Earnings guidance and market uncertainty, J. Accounting \& Economics 48 (2009), 90-109.

[RogW] L. C. G. Rogers, D. Williams, Diffusions, Markov processes and martingales, Vol. 1, second ed., Cambridge Univ. Press, Cambridge, 1994.

[Rom] D. H. Romer, Advanced Macroeconomics, 5th ed., McGraw-Hill, 2018.

[Rus] D. L. Russell, Mathematics of Finite-Dimensional Control Systems: Theory and Design, Lecture Notes Pure Appl. Math., Marcel Dekker, New York, 1979.

[Sch1] M. Schröder, Brownian excursions and Parisian barrier options: a note, J. Appl. Probab. 40 (2003), 855-864.

[Sch2] M. Schröder, Laguerre series in contingent claim valuation, with applications to Asian options, Math. Finance 15 (2005), 491-531.

[Sch3] M. Schröder, On ladder height densities and Laguerre series in the study of stochastic functionals, Adv. in Appl. Probab. 38 (2006), I: 969-994, II: 995-1027

[Sch4] M. Schröder, On arithmetic-average Asian power-options: closed forms and explicit methods for valuation, Quart. J. Mech. Appl. Math. 66 (2013), 1-27.

[Son] E. Sontag, Mathematical Control Theory: Deterministic Finite Dimensional Systems, second ed., Springer, New York, 1998.

[Var] H. R. Varian, Microeconomic Analysis, third ed., Norton, 1992. 